\title{
Estado Actual de las Políticas Públicas a Favor de las Pequeñas y Medianas Empresas en Cartagena y Estrategias Aplicables al Plan de Desarrollo Distrital 2020 - 2023
}

\author{
Current Status of Public Policies in Favor of Small and Medium-sized Enterprises in \\ Cartagena and Strategies Applicable to the District Development Plan 2020 - 2023 \\ ${ }^{a}$ Yeison Manuel Guzmán Vega ${ }^{9}$, Natalia Ramos de Ávila ${ }^{b}$ \\ a yeison.guzman2000@gmail.com “Semillero Políticas Públicas Participación y Desarrollo, Programa de Derecho, Universidad de \\ Cartagena. Cartagena, Colombia.

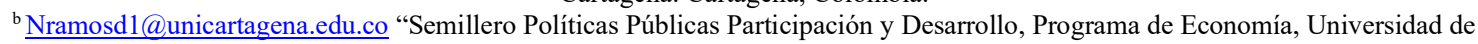 \\ Cartagena. Cartagena, Colombia.
}

\footnotetext{
Forma de Citar: Y.M. Guzmán -Vega y N. Ramos-De Ávila "Estado Actual de las Políticas Públicas a Favor de las Pequeñas y Medianas Empresas en Cartagena y Estrategias Aplicables al Plan de Desarrollo Distrital 2020 - 2023", Rev. Saberes, Vol. 13, No. 02, pp. $35-45,2020$.
}

Recibido: 24/03/2020 Evaluación: 28/05/2020 Aceptado: 30/06/2020 DOI: $\underline{\text { https://doi.org/1025213/1794-4384/1302.0005 }}$

\section{Resumen}

En la presente investigación se analizaron cuáles fueron las acciones implementadas por el Distrito de Cartagena para incrementar la cultura del emprendimiento en el plan de desarrollo (2016-2019); de esta manera, se logró identificar los errores cometidos en las políticas públicas establecidas en ese lapso y así mismo, aquellas propuestas tendientes a incrementar la creación de empresas ejecutadas de forma exitosa por algunas ciudades o países, obtenteniendo al final las estrategias pertinentes y eficaces para fomentar el emprendimiento formal en el plan de desarrollo (2020-2023).

\section{Palabras Clave}

Plan de desarrollo, emprendimiento, MCPyMES, políticas públicas.

\section{Abstract}

In this research we will analyze what were the actions implemented by the District of
Cartagena to increase the culture of entrepreneurship in the development plan (2016-2019); In this way, the mistakes made in the public policies established in that period will be identified and those proposals aimed at increasing the creation of companies executed successfully by some cities or countries will be studied, all this will be done in order to obtain the most relevant and effective to promote formal entrepreneurship in the development plan (2020-2023).

\section{Keywords}

Development plan, entrepreneurship, MCPyMES, public policies

\section{Introducción}

El 01 de enero de 2020 se posesiono como alcalde de Cartagena William Dautt Chamatt, los retos que debe cumplir el dignatario en su mandato Constitucional son variados, entre

\footnotetext{
${ }^{9}$ Autor para correspondencia: correo electrónico: yeison.guzman2000@gmail.com

C 2020 Fundación Universitaria Antonio de Arévalo - UNITECNAR. Este es un artículo bajo la licencia CC BY-NC-ND (http://creativecommons.org/licenses/by nc-nd/4.0/)
} 
ellos está, como lo establece la ley, ${ }^{10}$ redactar junto a su gabinete el plan de desarrollo para el periodo (2020-2023), el cual eventualmente será estudiado y decidido por parte del consejo distrital de la ciudad. Este proyecto establece las políticas públicas que se aplicaran durante los cuatro (4) años del mandato.

Antes de la realización del plan de desarrollo (2020-2023) y teniendo en cuenta la importancia que ha tenido el sector empresarial para el desarrollo económico de las ciudades (Delfín y acosta, 2016), se hace propicio realizar un análisis de las acciones implementadas por la administración distrital de Cartagena en la elaboración de las políticas públicas a favor de los pequeños y medianos empresarios en el periodo anterior, es decir, 2016-2019.

Lo anterior se realizará con el fin de identificar cuáles fueron las falencias del plan de desarrollo (2016-2019) en el área de emprendimiento y que aspectos pueden ser mejorados a través de propuestas realizables basadas en los estudios estadísticos y en las acciones de otros territorios a favor del emprendimiento.

Con el fin de abordar el tema descrito se desarrollaran los siguientes aspectos: 1 . Estudio del deber estatal de promover la creación de empresa y fortalecer el emprendimiento; 2 . Revisión, dentro del plan de desarrollo (2016-2019), de los ejes principales que han incluido las políticas públicas a favor de los pequeños y medianos empresarios en la ciudad de Cartagena; 3 . identificar algunas propuestas no incluidas en el plan de desarrollo y que eventualmente podrían ser aplicadas en el plan de desarrollo distrital; 4. Estudiar los beneficios y garantías ofrecidas a empresarios en otros territorios.

\section{Metodología}

Este trabajo es de tipo cualitativo-descriptivo, los datos fueron recolectados a través de una

\footnotetext{
${ }^{10}$ LEY 152 DE 1994 (Por la cual se establece la Ley Orgánica del Plan de Desarrollo)

${ }^{11}$ Por políticas y programas del Estado se entienden todas aquellas acciones de los gobiernos para influenciar en los
}

investigación documental de fuentes secundarias de información tales como: artículos de investigación, tesis y estadísticas publicadas por entidades oficiales, entre otros. El estudio se desarrollará a nivel teórico; En el abordaje del mismo se analizará a través de normas y sentencias el deber del Estado Colombiano de buscar estrategias que fomenten el emprendimiento. Luego, al analizar las políticas públicas de la ciudad (Cartagena) en el periodo 2016-2019 a favor de los pequeños y medianos empresarios se realizará una observación integra al plan de desarrollo en ese lapso. Por último, se identificarán las acciones desarrolladas en otras ciudades $\mathrm{o}$ países a favor del emprendimiento y las propuestas de la doctrina para incentivar la creación de medianas y pequeñas empresas lo cual, posiblemente, facilite la creación de políticas públicas pertinentes en el plan de desarrollo de Cartagena (2020-2023).

\section{Deber Estatal de Fomentar el Emprendimiento}

Desde antaño, las empresas han sido una parte importante de la sociedad pues estas traen consigo beneficios para el país en el cual funcionen, a saber: desarrollo económico, generar riquezas, desarrollar actividades productivas, desarrollar talentos, otorgar empleos a los ciudadanos. Todas estas se adaptan a las nuevas tecnologías con relativa facilidad entre otros aspectos positivos que se traducen, la mayoría de las veces, en mejora de la calidad de vida de los ciudadanos (Delfín y acosta, 2016).

Por las anteriores cualidades es necesario que el Estado desarrolle políticas públicas ${ }^{11}$ que fomenten la participación empresarial en el sentido de crear estrategias o alternativas que permitan al emprendedor ver atractivo iniciar su proyecto en el respectivo territorio, como lo sería, por ejemplo, que el Estado fomente jornadas de capacitación cuando se presenten

mecanismos de mercado con el fin de que funcionen eficientemente mediante la eliminación de fallas de mercado y regulaciones administrativas rígidas (Álvarez, 2009). 
imperfección en la información publicada, errores conocidos como fallas del mercado ${ }^{12}$ (Stam et al., 2006) y (Zambrano L. 2016).

En Colombia, la creación de estrategias que propendan por incentivar a los micro, pequeños y medianos empresarios (hoy conocidos por sus siglas como MIPYMES) es una obligación interpuesta por normas jurídicas que se extiende por toda la pirámide kelseniana, es decir, va desde la Constitución política y pasa por gran parte del ordenamiento jurídico colombiano. Su lugar en el régimen Constitucional lo ha señalado la Corte Constitucional de la siguiente manera:

"La Constitución de 1991 al adoptar un modelo de Estado Social de Derecho, introdujo un modelo de economía social de mercado en el que, de un lado, se admite que la empresa es motor de desarrollo social y por esta vía se reconoce la importancia de una economía de mercado y la promoción de la actividad empresarial, pero por otro, se asigna al Estado no sólo la facultad sino la obligación de intervenir en la economía con el fin de remediar las fallas del mercado y promover el desarrollo económico y social (C-263/11)".

De esta forma, la Constitución política ha establecido en su artículo 333 que el "Estado estimulara el desarrollo empresarial"; no obstante, como ha sido señalado por el tribunal Constitucional (C-263/11) "la intervención del Estado en la economía se lleva a cabo con la concurrencia de las ramas del poder público" es decir son la rama legislativa, ejecutiva, de manera principal, y la rama judicial, de manera segundaria, las que se encargan de cumplir el mandato constitucional estableciendo estrategias que incentiven fomentar el emprendimiento en el país.

En la tabla número uno (1) (ver Anexos, tabla \#1) se plasma de forma general algunas leyes,

\footnotetext{
12 Fallos de mercado, determinadas como: "todas aquellas situaciones en las que el mecanismo del mercado no puede funcionar adecuadamente" situación que tiene como consecuencia "invariablemente perjuicios para la sociedad, generalmente en términos de pérdida de valor", lo anterior ocasionando que el Estado deba intervenir en la economía (Rodríguez, 2013).
}

decretos y sentencias expedidas por las ramas del poder público que reiteran la obligación Estatal de tomar acciones tendientes a incentivar la creación de empresas:

\section{Estrategias en el plan de desarrollo para las MiPymes}

Como se vio en la ley 905 de 2004, (citadas en la tabla \#1), las entidades territoriales ${ }^{13}$ como pertenecientes al poder ejecutivo son responsables de fomentar $y$ dirigir el emprendimiento en el país, de tal perspectiva que Cartagena como Distrito Turístico y cultural, ubicada en el departamento de Bolívar-Colombia, debe implementar estrategias tendientes a incentivar a sus ciudadanos a la creación de empresas. La mayoría de las acciones tendientes a cumplir el deber Constitucional detallado anteriormente se establece en el plan de desarrollo de la ciudad.

El último plan distrital de desarrollo fue aprobado por el Consejo Distrital de Cartagena a través del Acuerdo No.006 el 13 de junio de 2016, el cual fue denominado "Primero la Gente para una Cartagena Sostenible y Competitiva" y presentado por quien en ese momento era alcalde de la ciudad, Manuel Vicente Duque Vásquez y aunque este renuncio a su cargo tras investigaciones por la elección irregular de la contralora Nubia Fontalvo $^{14}$ y en su lugar gobernaron varios alcaldes encargados, el plan de desarrollo siguió aplicándose.

Las estrategias de este plan de desarrollo a favor de los pequeños y medianos empresarios tienen su espacio en el artículo 9 del Plan de desarrollo denominado "adaptar el territorio para la gente”, específicamente en el eje titulado "desarrollo económico incluyente" y

\footnotetext{
${ }^{13}$ Son entidades territoriales (según librado Rodríguez 2017) los departamentos, los distritos, los municipios y los territorios indígenas.

14 Periódico "El Espectador", artículo titulado: "Renuncia Manuel Vicente Duque, suspendido alcalde de Cartagena" 31 Oct 2017 - 8:23 PM, recuperado de: https://www.elespectador.com/noticias/nacional/bolivar/renunc ia-manuel-vicente-duque-suspendido-alcalde-de-cartagenaarticulo-720871
} 
su línea estratégica denominada "Cartagena ciudad para invertir" que cuenta con 6 programas y 19 subprogramas (ver Anexos, tablas \#2, 3, 4, 5, 6 y 7 se detalla cada punto del eje).

Además de lo anterior, es importante señalar que en la ciudad se ha analizado un alto emprendimiento en los últimos años. Según estadísticas de la Cámara de Comercio de Cartagena, en el norte de Bolívar, para el año $2017^{15}$ se crearon aproximadamente 7.844 empresas; en el año 2018 se crearon 8.764 empresas lo cual significa un crecimiento del $12 \%$ respecto a las cifras del año anterior; además, para el año 2019, se observó la creación de 8.651 unidades empresariales, lo cual representa una disminución de un 1,3\% respecto al año anterior ${ }^{16}$.

Aunque se deben reconocer las cualidades positivas del proyecto; no obstante, sería erróneo llegar concluir que solo por la intervención del plan de desarrollo se ha generado el avance dado que esa afirmación conllevaría a lo que es denominado por los expertos en políticas públicas "sesgo por factores externos", ${ }^{17}$ pues para poder llegar a afirmaciones objetivas respecto a la relación entre políticas públicas y resultados (también llamados outcome), es necesario hacer un estudio entre lo que paso con la población a la cual se le aplico el programa y lo que hubiese pasado si la política no se implementara, lo que también se conoce en la doctrina como hipótesis contra-factual o estimación del contra-factual.

"El impacto $(\alpha)$ de un programa es la diferencia entre los resultados (Y) de la misma persona cuando ha participado y cuando no ha participado en el programa" (Gertler Paul, Martínez Sebastián, Premand Patrick, Rawlings Laura y Vermeersch Christel, 2011).

\footnotetext{
15 https://www.cccartagena.org.co/2019/05/13/crece-elemprendimiento-en-cartagena/

${ }_{16} \mathrm{https}: / / \mathrm{www} . c c c a r t a g e n a . o r g . c o / w p-$ content/uploads/2020/02/Informe-Econo $\%$ CC\%81 micoJurisdiccio $\%$ CC $\% 81 \mathrm{n}-\mathrm{Ca} \% \mathrm{CC} \% 81$ mara-de-Comercio-deCartagena-2019.pdf

${ }^{17}$ Atribuir al programa lo que en realidad es el efecto de otras cosas que están ocurriendo al mismo tiempo
}

Además de lo anterior, se observa que la aplicación del plan de desarrollo a mediado de 2019 solo era del 65\%, (se detalla adelante) y que según los rankings sobre ciudades con ambientes emprendedores no ubican a Cartagena entre sus primeros puestos. lo cual hace menos asertiva la afirmación.

Por último, Es relevante mencionar que el tema a tratar en las siguientes líneas no se centra en hacer un estudio sobre la eficacia de la política pública de emprendimiento en Cartagena, pues con ello la investigación se desviaría de los objetivos propuesto, más bien, se buscara identificar estrategias que ayuden en la elaboración de un eventual plan de desarrollo en la ciudad.

\section{Aspectos por Mejorar del Plan de Desarrollo 2016-2019}

1. Un eje importante en el sector empresarial y que no tiene de forma directa una política pública en el plan de desarrollo es la deserción empresarial, según el informe económicojurídico de la cámara de comercio de Cartagena $^{18}, 5321$ empresas fueron cerradas en 2019, lo cual es alarmante y exige estrategias para obtener empresas estables, pues de nada serviría preocuparnos tanto por la creación cuando la vida de las empresas es muy corta.

2. Sobre los avances en la implementación del plan de desarrollo en su línea a favor del emprendimiento, se encuentra que según la fundación cívica pro-Cartagena (funcicar) ${ }^{19}$ en el 2do año de implementación, solo se había desarrollado el $37 \%$ del mismo, además el lugar donde más se ejecutó la política pública fue en la localidad 1, parte de la ciudad que abarca los barrios de estratos más altos ${ }^{20}$; mientras que en la localidad 2, donde habitan

\footnotetext{
$18 \quad$ https://www.cccartagena.org.co/wpcontent/uploads/2020/02/Informe-Econo $\%$ CC $\% 81 \mathrm{mico-}$ Jurisdiccio $\%$ CC $\% 81$-Ca $\%$ CC $\% 81$ mara-de-Comercio-deCartagena-2019.pdf

19 Una Fundación que trabaja por el fortalecimiento de la democracia en Cartagena $y$ el Caribe colombiano. http://www.funcicar.org/conocenos/ 20 Ver: https://www.dadiscartagena.gov.co/images/docs/crue/localidad es/barrios_localidad1_2015.pdf
} 
los barrios más pobres ${ }^{21}$, el cumplimiento solo fue de un $20 \%$. Para el año $2018^{23}$, el cumplimiento del plan de desarrollo solo fue del 50\% y para 2019 el cumplimiento del plan fue de $65 \%$. Por lo cual, se hace necesario que el próximo plan sea realizable, además, se debe ampliar el área de aplicación a los barrios más pobre.

3. Uno de los problemas que afecta estructuralmente el emprendimiento en Cartagena es la informalidad, la cual no tiene una definición establecida, pero podríamos entenderla teniendo en cuenta a (Fernández Cristina, 2018) como: "las firmas (o empresas) que realizan actividades legales $y$ no se encuentran bajo la órbita del Estado", ejemplo de ello lo encontramos en aquellas empresas que no se han inscrito en la cámara de comercio respectiva y no pagan impuestos. Y aunque se esperan los resultados del censo empresarial 2019, realizado por la cámara de comercio de Cartagena en convenio con el Distrito para conocer de forma precisa el estado de la informalidad empresarial ${ }^{24}$, varias fuentes, no estadísticas ${ }^{25}$, nos muestran que en Cartagena y en general en Colombia hay alta informalidad empresarial (OIT, 2014) lo cual repercute de forma negativa para el fomento del emprendimiento en la ciudad, pues entre uno de los riesgos para las empresas informales que ha señalado la Cámara de Comercio de Bogotá (CCB) esta "No poder acceder a los beneficios que el gobierno genera para los empresarios ${ }^{26}$, , es decir, no pueden acceder a los beneficios de las políticas públicas.

Aunque en el plan de desarrollo analizado se implementó un subprograma, denominada "subprograma inclusión para la transformación de la informalidad" (ver tabla

\footnotetext{
${ }^{21}$ https://www.banrep.gov.co/es/pobreza-cartagena-analisisbarrios,

https://www.dadiscartagena.gov.co/images/docs/crue/localidad es/barrios_localidad2_2015.pdf

${ }^{22}$ Información tomada de: http://www.funcicar.org/conoce-vala-ejecucion-del-plan-desarrollo-localidad/ 23

https://www.cartagena.gov.co/images/2019/Rcuentas/info.pdf

24 Información dada por exsecretario de Hacienda Distrital, William Valderrama (ver: https://www.eluniversal.com.co/economica/censo-empresarialayudara-al-distrito-a-mejorar-su-gestion-tributaria-FF2337052)
}

\#3) que buscaba la reducción de la informalidad, es necesario que se amplié el área de acción a aspectos más grandes como lo sería un programa completo que tenga un presupuesto más alto para lograr que aquellos que no están registrados ni recibiendo las políticas públicas puedan gozar de estos beneficios.

4. Por último, se resalta que según los resultados arrojados por la segunda edición del Índice de Competitividad de Ciudades (ICC), elaborado por el Consejo Privado de Competitividad (CPC) y la Universidad del Rosario $^{27}$ en su pilar "entorno para los negocios", Cartagena se sitúa en la posición número 14 de 23 ciudades analizadas, quedando mejor posicionadas ciudades como Manizales, Pereira , Bogotá, Medellín, Montería, Cúcuta, Valledupar, Neiva, Quibdó, Armenia, Villavicencio, Sincelejo y Barranquilla, muchas de las cuales no cuentan con los privilegios que posee Cartagena por ser histórica y turística $\mathrm{y}$, sin embargo, logran crear un ambiente eficaz para los negocios, por lo cual Cartagena debe apostar por mejorar su ambiente para las pequeñas y micro empresas a través de políticas públicas sólidas $\mathrm{y}$ pertinentes.

\section{Posibles Estrategias para el Plan de Desarrollo (2020-2023)}

1. En el trabajo llevado a cabo por el Banco de desarrollo de América Latina en 2017 para el caso de Ecuador plantea que "el estado puede basar sus políticas públicas en 4 ejes i) fomentando la actitud emprendedora a temprana edad, ii) asesorando sobre la viabilidad de una idea empresarial, iii) apoyando la puesta en marcha de una idea y iv)

\footnotetext{
${ }^{25}$ Working paper No. 76 2018-11, fedesarrollo y https:/www.portafolio.co/negocios/empresas/empresasinformales-ayudar-a-su-crecimiento-es-mejor-que-combatirlas511916, https:/www.eluniversal.com.co/economica/hay-quebajar-la-informalidad-del-70-en-cartagena-camara-decomercio-208065-NSeu310386

26 https://www.ccb.org.co/Fortalezca-su-empresa/Temasdestacados/Formalice-su-empresa/Beneficios-de-laformalidad-y-riesgos-de-la-informalidad

${ }^{27}$ Motivados por aportar herramientas que permitan contar con políticas públicas de competitividad basadas en evidencia
} 
ayudando a un negocio recién constituido." (CAF, 2017).

Cabe destacar que según Global Entrepreneurship Monitor, entre los años 2012-2017, ${ }^{28}$ un país como Ecuador con niveles bajos de innovación y creación de empresas, ha venido avanzado estos últimos años y siendo el emprendimiento un objeto importante de estudio para muchos académicos.

Entre las políticas públicas, las estrategias para fomentar del emprendimiento a temprana edad alcanzaron un valor muy representativo. Este análisis nos muestra que "se puede aplicar politicas públicas encaminadas a la población de infancia y adolescencia con el objetivo de fomentar a temprana edad implementación de cátedras de innovación o ideas de negocios, estas a su vez tienen que ir acompañada de apoyo en contextos o lugares donde se puedan dar a conocer dichos emprendimientos".

Los planes de desarrollo en la costa caribe y las políticas públicas implementadas como estrategias para cumplir dicho plan no se llevan a cabo de la manera adecuada en las instituciones educativas públicas, además los centros educativos se han mostrado débiles en los últimos años debido a los problemas de corrupción $^{29}$, por lo cual sería propicio impulsar una cultura empresarial en Cartagena desde las instituciones públicas buscando que los estudiantes de los estratos bajos se capaciten de forma temprana para la creación de empresas.

2. Un estudio realizado para la ciudad de Bogotá, una de las ciudades con mayor índice de emprendimiento, concluye que la principal función de las Instituciones estatales debe ser la de "facilitar la creación, el desarrollo y la sostenibilidad de los emprendimientos en la ciudad a través de más y mejores programas de apoyo empresarial diseñados según las

\footnotetext{
$28 \mathrm{https} / /$ gemconsortium.org/report/jovenes-emprendedoresen-ecuador-2012-2017

${ }^{29}$ https://www.semana.com/educacion/articulo/corrupcion-en-

el-programa-de-alimentacion-escolar/579501

${ }^{30} \mathrm{https}: / /$ www.dinero.com/economia/articulo/pymescolombia/212958
}

dinámicas mismas y las necesidades de los emprendedores" (Cardozo Santiago, 2017) aspectos que serían muy importante implementar en Cartagena donde hay informalidad empresarial, pues si se facilita los requisitos para la creación de empresas es posible que además de la creación de nuevos proyectos, se reduzca la informalidad.

3. Es importante crear Estrategias enfocadas solamente en micro empresas pues las pequeñas empresas pueden ayudar a reducir la pobreza en estos países, puesto que generan gran cantidad de empleo, niveles adecuados de calidad de empleo y bajo costo de bienes y servicios utilizados por los pobres (Vanderberg, 2007), además, en Colombia representan casi el $99 \%$ del total de las empresas y general el 38\% del PIB nacional ${ }^{30}$. No obstante, según lo manifiesta (Ospina y Rodríguez, 2007).

"falta una mayor difusión de las políticas públicas para el fomento de la MIPyMEs, tanto en el ámbito nacional como departamental, que permita el conocimiento generalizado por parte de la comunidad".

Por lo cual se hace necesaria la implementación de planes tendientes a beneficiar las pequeñas y medianas empresas en Cartagena a través de políticas públicas.

4. Por último, es propicio reconocer la importancia de un programa implementado en Medellín durante la administración de Sergio fajardo (2004-2007) llamado, "programa de cultura E" el cual ha invertido seis mil millones de pesos en 220 a empresas de la región, como capital semilla de aquellos emprendimientos que cumplen una serie de requisitos de viabilidad y pertinencia. Estrategia que ha visto sus frutos en el crecimiento de las empresas en Medellín y que se pueden observar en los rankings de emprendimiento $^{31}$ y en el reconocimiento otorgado a la ciudad denominado líder

\footnotetext{
31 segunda edición del Índice de Competitividad de Ciudades (ICC), elaborado por el Consejo Privado de Competitividad (CPC) y la Universidad del Rosario en su pilar "entorno para los negocios"
} 
mundial en fomento y promoción de nuevos emprendedores, otorgado en el evento Emtech Colombia (2012).

\section{Conclusiones}

Se hace notorio que las políticas públicas establecidas en el plan de desarrollo (20162019) a favor de los MIPYMES en la ciudad de Cartagena, a pesar de incluir diversos temas pertinentes para fomentar el emprendimiento, estos deben incluir otros aspectos más conectados con las necesidades de tres grupos diferentes, por un lado; los actuales empresarios, por otro, aquellos que ejecutan sus negocios de manera informal y por último, quienes están a las puertas de crear establecimientos, tales aspectos relacionados con la parte financiera, legal y fiscal, con el objetivo de fomentar un ambiente de confianza y sostenibilidad, buscando de esta forma posicionar a la ciudad de Cartagena con altos índices de productividad y desarrollo en toda Colombia, gracias al producto empresarial.

Por otra parte, es necesario que las políticas públicas establecidas en el plan de desarrollo de Cartagena (2020-2023) a favor del emprendimiento, puedan centrarse en aquellos puntos de la ciudad de estrato bajo que necesiten superar temas como la pobreza, la violencia, el analfabetismo, entre otros, planteando estrategias realizables y eficaces, como aquellas implementadas en otras ciudades que hoy son referente mundial de emprendimiento y desarrollo, (por ejemplo: Medellín-Colombia).

\section{Referencias Bibliográficas}

Cardozo Santiago B. $\begin{array}{r}(2017) \\ \text { estrategias }\end{array}$
gubernamentales para potenciar el
emprendimiento en Bogotá y facilitar su éxito
y contribución al desarrollo económico de la
ciudad" (tesis de pregrado), Universidad
Colegio Mayor de Nuestra Señora del Rosario,
Bogotá-Colombia.

Congreso de Colombia. (2 de agosto de 2004) Ley 905 de 2004: Diario Oficial No. 45.628 (recuperado de: http://www.secretariasenado.gov.co/senado/b asedoc/ley 0905 2004.html).

Congreso de Colombia. (12 de julio 2000) Ley 590 de 2000. Diario Oficial No. 44.078 (recuperado de: http://www.secretariasenado.gov.co/senado/b asedoc/ley 0590 2000.html).

Corte Constitucional de Colombia, 6 de abril de 2011, Sentencia C-263/11, MP. JORGE IGNACIO PRETELT CHALJUB.

Consejo Distrital de Cartagena (13 de junio 2016) Acuerdo No. 006 de 2016. Plan de Desarrollo Distrital 2016 - 2019 "primero la gente para una Cartagena sostenible y competitiva" recuperado de: https://www.cartagena.gov.co/Documentos/2 016/Transparencia y aip/Plan de Desarrollo /2016/ACUERDO-006-2016-PLAN-DEDESARROLLO.pdf

Constitución política de Colombia [Const.] (1991) Artículo 13 [Titulo II]. 2da Ed. Legis.

Delfín L.y Acosta M, 2016. "Importancia y análisis del desarrollo empresarial" Revista científica Pensamiento y Gestión, N40: Ene Jun 2016, universidad del norte (BarranquillaColombia).

Fernández C. (2018), "Informalidad empresarial en Colombia" Working paper No. 76 2018-11. Centro de investigación económica social, fedesarrollo.

Gertler Paul J, Martínez Sebastián, Premand Patrick, Rawlings Laura B., VermeerschC hristel M. J. (2011). Cap. "Inferencia causal y contrafactuales". Libro: La evaluación de impacto en la práctica (P.P. 33-46). Washington DC. Banco Mundial.

Orozco Triana, J., \&amp; Arraut Camargo, L. (2013). "El entorno competitivo para el emprendimiento en la región Caribe de Colombia: caso de Barranquilla, Cartagena, Santa Marta y Sincelejo". Revista Escuela De Administración De Negocios, (74), 86-105. 
https://doi.org/10.21158/01208160.n74.2013. $\underline{738}$

Ospina D. L. (2007) "Políticas públicas de micro, pequeñas y medianas empresas en el departamento de caldas" revista jurídica Universidad de Caldas. Manizales (Colombia).

Organización internacional del trabajo, (2014). "Políticas para la formalización de las micro y pequeñas Empresas en Colombia". Programa de promoción de la formalización FORLAC. Recuperado de: https:/www.ilo.org/wcmsp5/groups/public/--americas/---ro-

lima/documents/publication/wcms 318206.p $\underline{\mathrm{df}}$

Presidente de la República de Colombia, (junio 16 1971) Decreto 410 de 1971: Cód. De Comercio, Diario oficial: 33.339 (recuperado de:

https://www.alcaldiabogota.gov.co/sisjur/nor mas/Norma1.jsp? $i=41102$ ).

Rodríguez C. E. (2013). "Las fallas del mercado". "fallas de mercado y regulación económica: ¿la regulación ejercida por el gobierno permite lograr un mejor funcionamiento de los mercados?" VL - 21 DO - 10.15381/quipu.v21i39.6800

Rodríguez L. R. 2017, DERECHO ADMINISTRATIVO GENERAL Y COLOMBIANO Tomo I, EDITORIAL TEMIS S. A. Bogotá - Colombia Vandenberg, P. (2007). Poverty reduction through small enterprises Emerging consensus, unresolved issues and ILO activities. Small Enterprise Development Programme Job Creation and Enterprise Development Department International Labour Office · Geneva.

Zambrano L. (2016) "El papel del Estado en el desarrollo del emprendimiento: Comparativo Colombia - Ecuador", (trabajo de maestría), universidad nacional de Colombia. (http://bdigital.unal.edu.co/57213/7/luzadrian azambranocarbonell.2016.pdf) 
Saberes, II - 2020, ISSN: 1794 -4384 (En línea), 1794 -4384 (Impreso)

Anexos:

Tabla 1: Normas y sentencias a favor de emprendedores

\begin{tabular}{|c|l|}
\hline Fuente legal o jurídica & \multicolumn{1}{|c|}{ Contenido } \\
\hline $\begin{array}{c}\text { Art 333 de la Constitución política de } \\
\text { Colombia (1991) }\end{array}$ & $\begin{array}{l}\text { "El Estado fortalecerá las organizaciones solidarias y } \\
\text { estimulará el desarrollo empresarial". }\end{array}$ \\
\hline Ley 590 de 2000 & $\begin{array}{l}\text { "Por la cual se dictan disposiciones para promover el } \\
\text { desarrollo de las micro, pequeñas y medianas empresa". }\end{array}$ \\
\hline Ley 905 de 2004 & $\begin{array}{l}\text { responsabiliza a las entidades territoriales a promover las } \\
\text { MiPymes, reestructura los principales organismos } \\
\text { encargados de direccionar el emprendimiento en el pías }\end{array}$ \\
\hline Decreto 410 de 1971: Cód. del & $\begin{array}{l}\text { por el cual se regula la actividad comercial del país y se } \\
\text { establece cual es la ley y el juez competente para la } \\
\text { actividad comercial, aspecto en el cual se suscriben las } \\
\text { empresas }\end{array}$ \\
\hline Sentencia C-263/11 & $\begin{array}{l}\text { "se admite que la empresa es motor de desarrollo social y } \\
\text { (..) se le asigna al Estado la facultad y la obligación de } \\
\text { intervenir en la economía con el fin de remediar las fallas } \\
\text { del mercado y promover el desarrollo económico y social" }\end{array}$ \\
\hline
\end{tabular}

Nota: elaboración propia con base en la norma

Tabla 2: Plan de desarrollo 2016-2017 programa 1

\begin{tabular}{|c|c|c|}
\hline PROGRAMA & SUBPROGRAMA & ESTRATEGIAS \\
\hline \multirow{2}{*}{$\begin{array}{l}\text { 1. PROGRAMA CARTAGENA } \\
\text { CIUDAD PARA INVERTIR (objetivo: } \\
\text { fortalecer la competitividad local y el } \\
\text { desarrollo económico) } \\
\text { (encargado: secretaria de hacienda } \\
\text { Distrital y secretaria general) }\end{array}$} & $\begin{array}{l}\text { SUBPROGRAMA FORTALECIMIENTO } \\
\text { INSTITUCIONAL PARA LA } \\
\text { COMPETITIVIDAD Y CRECIMIENTO } \\
\text { ECONOMCO }\end{array}$ & $\begin{array}{l}\text { Busca que a través de la creación de Centros de Desarrollo } \\
\text { Cultura, la empresa innove en los productos, servicios Etc. } \\
\text { que generen beneficios directos a la ciudadanía, la economía } \\
\text { naranja y fortalecimiento de la cultura }\end{array}$ \\
\hline & $\begin{array}{c}\text { SUBPROGRAMA CON CIENCIA, } \\
\text { TECNOLOGIA E INNOVACION CARTAGENA } \\
\text { VA }\end{array}$ & $\begin{array}{l}\text { Se apoyarán las iniciativas que fortalezcan la relación } \\
\text { Universidad - Empresa - Estado, como Una estrategia para } \\
\text { promover la investigación, el degarrollo tecnológico y la } \\
\text { imovación. Igualmente se aprovecharán las politicas públicas } \\
\text { del gobierno nacional que fortalezcan las condiciones de } \\
\text { proyectos de ciencia, tecnologia e innovación }\end{array}$ \\
\hline
\end{tabular}

Nota: elaboración propia con base en el Plan de Desarrollo Distrital " Primero la Gente, Para una Cartagena Competitiva y sostenible 2016-2019.

Tabla 3: Plan de desarrollo 2016-2019 programa 2

\begin{tabular}{|c|c|c|}
\hline $\begin{array}{l}\text { 2.PROGRAMA EMPLEO, INGRESO, } \\
\text { EMPRENDIMIENTO }\end{array}$ & $\begin{array}{l}\text { SUBPROGRAMA INCLUSIÓN PARA } \\
\text { LA TRANSFORMACIÓN DE LA } \\
\text { INFORMALIDAD }\end{array}$ & $\begin{array}{l}\text { Busca disminuir la informalidad a través de 1capacitacion para organizar su negocio } \\
2 \text { reubicación en espacio público legalmente a través de una contraprestación } \\
\text { económica, } 3 \text { selección de proyectos para entregar capital semilla }\end{array}$ \\
\hline $\begin{array}{l}\text { (objetivo: mitigar el desempleo } \\
\text { capacitando, con alianzas con el sector } \\
\text { privado y entrega de créditos y }\end{array}$ & $\begin{array}{l}\text { SUBPROGRAMA PRIMERO TÚ } \\
\text { MUJER CON AUTONOMIA } \\
\text { ECONÓMICA }\end{array}$ & $\begin{array}{l}\text { Para promover los derechos económicos de las mujeres, se realizarán acciones de } \\
\text { promoción de emprendimientos en } \\
\text { Áreas de desarrollo, en los mercados y sus oportunidades. }\end{array}$ \\
\hline $\begin{array}{l}\text { microcréditos como capital semilla) } \\
\text { (encargados: Gerencia de Espacio } \\
\text { Público; Secretaria de Participación }\end{array}$ & $\begin{array}{l}\text { SUBPROGRAMA GENTE JOVEN Y } \\
\text { EMPRENDEDORA }\end{array}$ & $\begin{array}{l}\text { Se proporcionará a los jóvenes la inclusión en el mercado, en el marco del Sistema } \\
\text { de intermediación laboral con las agencias públicas de empleo y } \\
\text { generación de ingresos }\end{array}$ \\
\hline $\begin{array}{l}\text { Unidad de Juventud; Secretaria del } \\
\text { Interior y Convivencia Ciudadana }\end{array}$ & $\begin{array}{c}\text { SUBPROGRAMA } \\
\text { EMPRENDIMIENTO ÉTNICO }\end{array}$ & $\begin{array}{l}\text { Se desarrollarán estrategias de articulación que permita aprovechar (a las } \\
\text { poblaciones étnicas) las ventajas de Cartagena, como sitio de predilección turística. }\end{array}$ \\
\hline
\end{tabular}

Nota: elaboración propia con base en el Plan de Desarrollo Distrital " Primero la Gente, Para una Cartagena Competitiva y sostenible 2016-2019. 
Saberes, II - 2020, ISSN: 1794 -4384 (En línea), 1794 -4384 (Impreso)

Tabla 4: Plan de desarrollo 2016-2019 programa 3

\begin{tabular}{|c|c|c|}
\hline \multirow{5}{*}{$\begin{array}{l}\text { 3.PROGRAMA TURISMO } \\
\text { COMPETITIVO Y FUENTE DE } \\
\text { DESARROLLO SOSTENIBLE } \\
\text { (Objetivo: posicionar a Cartagena como } \\
\text { destino turistico competitivo y fuente de } \\
\text { desarrollo sostenible. Fomento de } \\
\text { alianzas público-privadas, canalizando } \\
\text { recursos de las regalias y la creación de } \\
\text { una dependencia distrital para vigilar el } \\
\text { turismo) } \\
\text { (responsable: CORPOTURISMO) }\end{array}$} & $\begin{array}{l}\text { SUBPROGRAMA NUEVA Y } \\
\text { VARIADA OFERTA TURISTIICA }\end{array}$ & $\begin{array}{l}\text { Propenderá por facilitar la creación de nuevos productos turisticos e identificar } \\
\text { circuitos que amplien la oferta y permita a los visitantes tener acceso a más opciones } \\
\text { de actividades, buscando que se queden más tiempo y haya intercambio cultural. }\end{array}$ \\
\hline & $\begin{array}{l}\text { SUBPROGRAMA CARTAGENA } \\
\text { DESTINO TURÍSTICO SOSTENIBLE } \\
\text { Y DE CALIDAD }\end{array}$ & $\begin{array}{l}\text { Se busca prestar servicios turisticos bajo estándares internacionales y sostenibles } \\
\text { financieramente para ello, se trabajará en la cualificación del talento humano } \\
\text { organizaciones del sector y fortalecimiento de productos que hoy se ofrecen }\end{array}$ \\
\hline & $\begin{array}{l}\text { SUBPROGRAMA APERTURA Y } \\
\text { CONSOLIDACIÓN DE MERCADOS } \\
\text { EMISORES ESTRATÉGICOS A } \\
\text { NIVEL NACIONAL E } \\
\text { INTERNACIONAL }\end{array}$ & $\begin{array}{l}\text { Busca seguir posicionando a Cartagena como destino turístico de alto nivel, para ello } \\
\text { se focalizarán las acciones de promoción en: productos y experiencias, además se } \\
\text { desarrollará ferias, entre otros similares. }\end{array}$ \\
\hline & $\begin{array}{l}\text { SUBPROGRAMA TURISMO } \\
\text { RESPONSABLE E INCLUYENTE }\end{array}$ & $\begin{array}{l}\text { Busca mejorar las condiciones de las comunidades locales poniendo en valor a } \\
\text { oferta de servicios turisticos. Además, buscara que el habitante conozca la ciudad }\end{array}$ \\
\hline & $\begin{array}{l}\text { SUBPROGRAMA CARTAGENA MÁS } \\
\text { CONECTADA }\end{array}$ & $\begin{array}{l}\text { Busca que el turista tenga facilidad aérea y terrestre para llega a la ciudad para ello } \\
\text { identificarán las rutas y aerolineas con las que mejor se pueda conectar. }\end{array}$ \\
\hline
\end{tabular}

Nota: elaboración propia con base en el Plan de Desarrollo Distrital " Primero la Gente, Para una Cartagena Competitiva y sostenible 2016-2019.

Tabla 5: Plan de desarrollo 2016-2019 programa 4

\begin{tabular}{|c|c|l|}
\hline \begin{tabular}{c} 
4. PROGRAMA \\
INTEGRACIÓN \\
TERRIORIAL \\
(objetivo: integración del \\
área regional del Distrito \\
\cline { 2 - 3 } de Cartagena)
\end{tabular} & $\begin{array}{c}\text { SUBPROGRAMA PROYECTOS } \\
\text { ENTRE CIUDADES }\end{array}$ & $\begin{array}{l}\text { Se formularán proyectos conjuntos con otros municipios de } \\
\text { beneficio común para un desarrollo sostenible y } \\
\text { competitivo. }\end{array}$ \\
\cline { 2 - 3 }
\end{tabular}

Nota: elaboración propia con base en el Plan de Desarrollo Distrital " Primero la Gente, Para una Cartagena Competitiva y sostenible 2016-2019.

Tabla 6: Plan de desarrollo 2016-2019 programa 5

\begin{tabular}{|c|c|l|}
\hline 5. PROGRAMA & SUBPROGRAMA & \\
MERCADOS PRIMERO LA & COMERCIANTES DE LOS & Busca mejorar las condiciones de higiene, aseo y \\
GENTE & MERCADOS PÚBLICOS & salubridad en el mercado y formalizar a los comerciantes. \\
(Objetivo: fortalecerá la red & DE CARTAGENA CON \\
de mercados para el Distrito & CONDICIONES MEJORADAS & \\
\cline { 2 - 3 } $\begin{array}{c}\text { de Cartagena, a través de la } \\
\text { dotación del mercado de } \\
\text { Santa-Rita y la formalización } \\
\text { de los comerciantes) }\end{array}$ & SUBPROGRAMA RED DE & $\begin{array}{l}\text { Su propósito es poner en funcionamiento el mercado de } \\
\text { Santa Rita, organización del traslado del mercado de } \\
\text { Bazurto y la construcción de la central de abastos. }\end{array}$ \\
\hline
\end{tabular}

Nota: elaboración propia con base en el Plan de Desarrollo Distrital " Primero la Gente, Para una Cartagena Competitiva y sostenible 2016-2019. 
Saberes, II - 2020, ISSN: 1794 -4384 (En línea), 1794 -4384 (Impreso)

Tabla 7: Plan de Desarrollo 2016-2019 programa 6

\begin{tabular}{|c|c|c|}
\hline \multirow{4}{*}{$\begin{array}{c}\text { 6. PROGRAMA } \\
\text { REACTIVACIÓN DEL } \\
\text { CAMPO } \\
\text { PARA LA } \\
\text { COMPETITIVIDAD } \\
\text { (Objetivo: creará un ambiente } \\
\text { favorable para impulsar las } \\
\text { inversiones agropecuarias y } \\
\text { pesqueras) }\end{array}$} & $\begin{array}{c}\text { SUBPROGRAMA } \\
\text { REACTIVACIÓN AGRICOLA } \\
\text { PARA LA GENTE }\end{array}$ & $\begin{array}{l}\text { E1 distrito buscara aumentar los ingresos de los MCPYME } \\
\text { empresarios del sector agricola, a través de tecnologías, } \\
\text { maquinas entre otros. }\end{array}$ \\
\hline & $\begin{array}{c}\text { SUBPROGRAMA } \\
\text { REACTIVACIÓN SOSTENIBLE, } \\
\text { DEL SECTOR PECUARIO PARA } \\
\text { LA GENTE }\end{array}$ & $\begin{array}{l}\text { Se identifican, delimitan y protegen las áreas agropecuarias } \\
\text { con potencial productivo para la actividad agropecuaria y } \\
\text { se establecerán canales de comercialización para los } \\
\text { productores pecuarios de distrito de Cartagena }\end{array}$ \\
\hline & $\begin{array}{c}\text { SUBPROGRAMA } \\
\text { SOSTENIBILIDAD DE LA } \\
\text { PESCA Y ACUICULTURA } \\
\text { PARA LA GENTE }\end{array}$ & $\begin{array}{l}\text { se amplia de manera sostenible y ecológica los niveles de } \\
\text { producción pesquera y agrícola para el aumento de los } \\
\text { ingresos de los pequeños y medianos pescadores y, } \\
\text { acuicultores artesanales }\end{array}$ \\
\hline & $\begin{array}{c}\text { SUBPROGRAMA } \\
\text { ASOCIACIONES RURALES } \\
\text { ORGANIZADAS Y } \\
\text { FORTALECIDAS PARA LA } \\
\text { PRODUCCIÓN AGROPECUARIA } \\
\text { DIVERSIFICADA Y } \\
\text { COMPETITIVA }\end{array}$ & $\begin{array}{l}\text { Se trabaja en el fortalecimiento de organizaciones } \\
\text { legalmente constituidas, para lograr una producción de } \\
\text { sector primario, sostenible y competitivo }\end{array}$ \\
\hline
\end{tabular}

Nota: elaboración propia con base en el Plan de Desarrollo Distrital " Primero la Gente, Para una Cartagena Competitiva y sostenible 2016-2019. 